\title{
Enhanced Axonal Metabolism during Early Natalizumab Treatment in Relapsing-Remitting Multiple Sclerosis
}

\author{
O.T. Wiebenga, A.M. Klauser, M.M. Schoonheim, G.J.A. Nagtegaal, M.D. Steenwijk, J.A. van Rossum, C.H. Polman,
}

F. Barkhof, P.J.W. Pouwels, and J.J.G. Geurts

\begin{abstract}
BACKGROUND AND PURPOSE: The considerable clinical effect of natalizumab in patients with relapsing-remitting multiple sclerosis might be explained by its possible beneficial effect on axonal functioning. In this longitudinal study, the effect of natalizumab on absolute concentrations of total $\mathrm{N}$-acetylaspartate, a marker for neuronal integrity, and other brain metabolites is investigated in patients with relapsing-remitting multiple sclerosis by using MR spectroscopic imaging.
\end{abstract}

MATERIALS AND METHODS: In this explorative observational study, 25 patients with relapsing-remitting multiple sclerosis initiating natalizumab treatment were included and scanned every 6 months for 18 months. Additionally 18 matched patients with relapsingremitting multiple sclerosis continuing treatment with interferon- $\beta$ or glatiramer acetate were included along with 12 healthy controls. Imaging included short TE 2D-MR spectroscopic imaging with absolute metabolite quantification of total $N$-acetylaspartate, creatine and phosphocreatine, choline-containing compounds, myo-inositol, and glutamate. Concentrations were determined for lesional white matter, normal-appearing white matter, and gray matter.

RESULTS: At baseline in both patient groups, lower concentrations of total $\mathrm{N}$-acetylaspartate and creatine and phosphocreatine were found in lesional white matter compared with normal-appearing white matter and additionally lower glutamate in lesional white matter of patients receiving natalizumab. In those patients, a significant yearly metabolite increase was found for lesional white matter total $\mathrm{N}$-acetylaspartate $(7 \%, P<.001)$, creatine and phosphocreatine $(6 \%, P=.042)$, and glutamate $(10 \%, P=.028)$, while lesion volumes did not change. In patients receiving interferon- $\beta$ /glatiramer acetate, no significant change was measured in lesional white matter for any metabolite, while whole-brain normalized lesion volumes increased.

CONCLUSIONS: Patients treated with natalizumab showed an increase in total $N$-acetylaspartate, creatine and phosphocreatine, and glutamate in lesional white matter. These increasing metabolite concentrations might be a sign of enhanced axonal metabolism.

ABBREVIATIONS: $\mathrm{GA}=$ glatiramer acetate; Glu = glutamate; IFNb = interferon $\beta$; LWM = lesional white matter; NAWM = normal-appearing white matter; MRSI = MR spectroscopic imaging; PVE = partial volume estimation; $\mathrm{tCr}=$ total creatine (creatine and phosphocreatine); tNAA = total $\mathrm{N}$-acetylaspartate; RRMS = relapsing-remitting multiple sclerosis

M ultiple sclerosis is an inflammatory and neurodegenerative disease of the central nervous system. The acute pathology of the disease is characterized by focal lesions in the white matter, ${ }^{1}$ while accumulation of gray matter damage is more prominent in the progressive stage of the disease. ${ }^{2}$

Previous spectroscopy studies found that lesional WM (LWM) (ie, only lesions or WM directly surrounding lesions) is

Received September 11, 2014; accepted after revision December 19.

From the Departments of Radiology and Nuclear Medicine (O.T.W., G.J.A.N. ${ }^{\dagger}$, M.D.S., F.B.), Anatomy and Neurosciences (O.T.W., A.M.K., M.M.S., G.J.A.N.' ${ }^{\dagger}$,.J.G.G.), Neurology (J.A.v.R., C.H.P.), and Physics and Medical Technology (P.J.W.P.), Neuroscience Campus Amsterdam and VU University Medical Center, Amsterdam, the Netherlands.

${ }^{\dagger}$ Deceased.

O.T. Wiebenga and A.M. Klauser contributed equally to this article. characterized by decreased levels of total $N$-acetylaspartate (tNAA), a marker of neuronal integrity, and decreased total creatine ( $\mathrm{tCr}$ ), a marker of energy metabolism, combined with higher levels of choline-containing compounds, a marker of cell membrane turnover. ${ }^{3,4}$ In the $\mathrm{WM}$, an increased $\mathrm{Cho} / \mathrm{tCr}$ ratio has been detected before the lesions are visible on conventional

This work was sponsored by the Dutch MS Research Foundation, grant number 09-358d. MR imaging was partly sponsored by Biogen Idec. Biogen Idec did not participate in any aspect of the design or performance (including data collection, data management, data analysis and interpretation, or preparation) of this investigator initiated study. Final approval for the work and manuscript was given by the authors.

Please address correspondence to Oliver T. Wiebenga, MD, VU University Medical Center, Department of Radiology and Nuclear Medicine, PO Box 7057, 1007 MB, Amsterdam, the Netherlands; e-mail: o.wiebenga@vumc.nl

$\equiv$ Indicates article with supplemental on-line appendix.

http://dx.doi.org/10.3174/ajnr.A4252 
Table 1: Baseline variables of patients and controls ${ }^{a}$

\begin{tabular}{lcccc}
\hline & $\begin{array}{c}\text { Natalizumab } \\
(\boldsymbol{n}=\mathbf{2 5})\end{array}$ & $\begin{array}{c}\text { IFNb/GA } \\
(\boldsymbol{n}=18)\end{array}$ & $\begin{array}{c}\text { Healthy } \\
\text { Controls } \\
(\boldsymbol{n}=\mathbf{1 2})\end{array}$ & $\boldsymbol{P}$ Value \\
\hline Age (yr) & $36.0 \pm 8.9$ & $38.2 \pm 5.0$ & $37.6 \pm 8.7$ & .619 \\
Sex (male/female) & $9: 16$ & $9: 9$ & $3: 9$ & .368 \\
EDSS & $3.0(1.5-6.5)$ & $2.5(1.0-6.5)$ & & .589 \\
Duration since onset (yr) & $7.9 \pm 6.1$ & $8.8 \pm 5.3$ & & .550 \\
Prior IFNb/GA duration at baseline (yr) & $2.6 \pm 3.0$ & $4.4 \pm 3.9$ & & .121 \\
Brain volumes & & & & \\
$\quad$ NGMV (L) & $0.75 \pm 0.04$ & $0.73 \pm 0.05$ & $0.77 \pm 0.04$ & .063 \\
$\quad$ NWMV (L) & $0.70 \pm 0.03$ & $0.69 \pm 0.03$ & $0.72 \pm 0.04$ & .061 \\
$\quad$ NBV (L) & $1.45 \pm 0.05$ & $1.42 \pm 0.08$ & $1.49 \pm 0.07$ & $.020^{\mathrm{d}}$ \\
T2 lesion volume $(\mathrm{mL})^{\mathrm{e}}$ & $6.1(2.4-14.3)$ & $4.9(2.4-11.9)$ & & .599 \\
\hline
\end{tabular}

Note:-EDSS indicates Expanded Disability Status Scale; NGMV, normalized total GM volume; NWMV, normalized total WM volume; NBV, normalized whole-brain volume.

${ }^{a}$ Data are mean $\pm S D$. When normally distributed, a multivariate general linear model was used with age and sex included as covariates. Nonparametric testing was performed using Kruskal-Wallis and post hoc Mann-Whitney $U$ tests. ${ }^{\mathrm{b}} \chi^{2}$ test.

${ }^{c}$ Median and range.

${ }^{\mathrm{d}}$ Only significant between patients treated with IFNb/GA and healthy controls.

${ }^{\mathrm{e}}$ Median and interquartile range.

MR imaging. ${ }^{5}$ In the normal-appearing WM (NAWM), decreased tNAA and increased mIns, a marker of gliosis, have been found $^{6-8}$ and have shown correlations with clinical disability scores. ${ }^{9,10}$

The anti-inflammatory effect of natalizumab, ${ }^{11}$ second-line therapy for relapsing-remitting MS (RRMS) in most countries, leads to a dramatic reduction in the formation of WM lesions, a decrease in the whole-brain atrophy rate, and improved rates of clinical progression and relapses. ${ }^{12,13}$ Previous studies have shown that interferon $\beta$-1b (IFNb) and glatiramer acetate (GA) increase tNAA concentrations in MS, ${ }^{14,15}$ but the effect of natalizumab remains unclear.

We, therefore, performed MR spectroscopic imaging (MRSI) in patients starting natalizumab treatment and repeated measurements every 6 months in LWM, NAWM, and GM. Measurements were compared with those in healthy controls. Additionally, patients with RRMS continuing on IFNb/GA were followed during the same period.

\section{MATERIALS AND METHODS \\ Subjects}

Three groups of subjects were investigated in this observational study: patients with RRMS starting natalizumab at baseline, those continuing IFNb/GA, and healthy controls (Table 1), as described separately (O.T.W. et al, unpublished data, 2015). The study was approved by the ethics review board of the VU University Medical Center, and all subjects gave written informed consent before participation.

Inclusion criteria for the study were an age between 18 and 65 years and a diagnosis of clinically definite MS (for patients only). ${ }^{16}$ Exclusion criteria were a history of psychiatric or neurologic disease (other than MS for patients), contraindications for MR imaging, and a history of alcohol or drug abuse.

Patients starting natalizumab $(n=25 ; 300 \mathrm{mg}$ intravenously every 4 weeks) had at least 1 prior period of IFNb/GA treatment with breakthrough disease or rapidly evolving active RRMS before starting treatment. These patients were included after the decision to start natalizumab had already been made. At baseline,
6 patients were treatment-naïve for natalizumab, 14 patients had received 1 , and 3 patients had received 2 infusions. Two patients who had already received 6 infusions when they joined the study were only analyzed at the 6-month visit and onward. All patients continued natalizumab treatment for the duration of the study.

Patients receiving IFNb/GA $(n=18)$ continued this treatment at baseline. Twelve patients used IFN-b, the dose and route of administration dependent on type, and 6 patients used GA, $20 \mathrm{mg}$ subcutaneously, daily. Patients receiving IFNb/GA were matched to those receiving natalizumab for age, sex, disability (Expanded Disability Status Scale), and duration of therapy. Two patients discontinued IFNb/GA treatment but remained in the study. One discontinued GA after baseline because of side effects (necrosis and scarring of skin at injection sites) combined with stable disease; another discontinued IFNb at month 12 due to conversion to secondary-progressive MS.

Healthy controls $(n=12)$ were age- and sex-matched to patients receiving natalizumab at baseline.

\section{MR Imaging Acquisition}

MR imaging was performed on a $1.5 \mathrm{~T}$ whole-body scanner (Sonata; Siemens, Erlangen, Germany) with an 8-channel receiver head coil. Sequences included a 3D T1-weighted MPRAGE sequence (TR, $2700 \mathrm{~ms}$; TE, $5 \mathrm{~ms}$; TI, $950 \mathrm{~ms} ; 176$ sagittal sections; 1.3 -mm section thickness; $1.3 \times 1.3 \mathrm{~mm}^{2}$ in-plane resolution), and a proton-attenuation/T2-weighted turbo spin-echo sequence (TR, $3130 \mathrm{~ms}$; TE, 24 and $85 \mathrm{~ms}$; 46 contiguous 3-mm axial sections; $1 \times 1 \mathrm{~mm}^{2}$ in-plane, aligned to the pituitary-fastigium line as outlined in Fig $1 A$ ).

2D-MRSI included a point-resolved spectroscopy sequence (TR/TE, 3000/30 ms) on a single 15-mm slab aligned to the sections of the proton-attenuation/T2 sequence, with the center touching the top of the corpus callosum. ${ }^{17}$ Depending on head size, the FOV was $160 \times 160$ or $140 \times 160 \mathrm{~mm}$ and the corresponding volume of interest was $70 \times 100$ or $80 \times 100 \mathrm{~mm}$. The use of $16 \times 16$ phase-encodings resulted in a voxel size of 1.3 or $1.5 \mathrm{~mL}$. Reference $2 \mathrm{D}$-MRSI datasets without water suppression were measured by using both head and body coils as a receiver.

\section{Brain and Lesion Volumes}

T2-hyperintense WM lesions were quantified by using an automated segmentation method. ${ }^{18}$ Normalized whole-brain WM and GM volumes were computed by using the 3D-T1 images and SIENAX (part of fMRI of the Brain Software Library 5.02; http:// www.fmrib.ox.ac.uk/fsl) after lesion filling, by using automated lesion-filling (Lesion Automated Preprocessing). ${ }^{19}$ The partial volume segmentation masks of WM, GM, CSF, and lesions, produced by SIENAX, was summed to compute a partial volume 

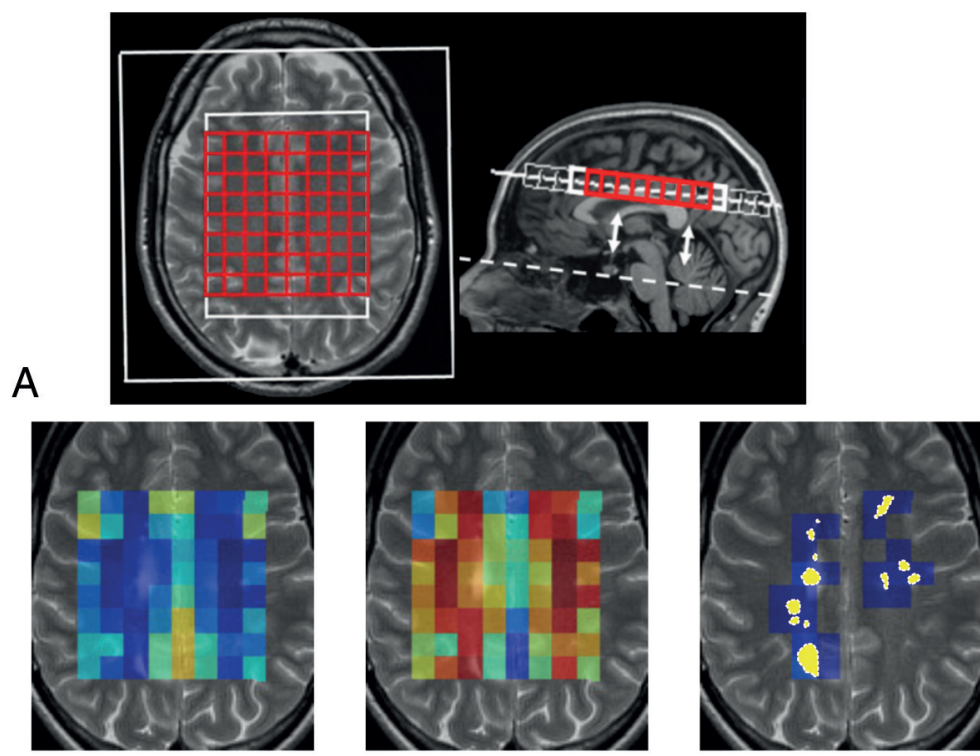

WM PVE

B

GM PVE

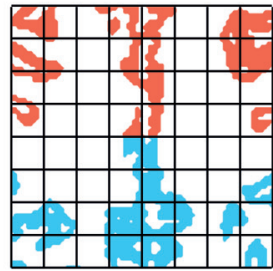

GM frontal / parietal C

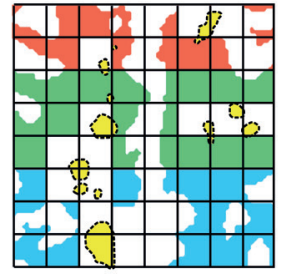

NAWM frontal / semioval / parietal
Lesions PVE

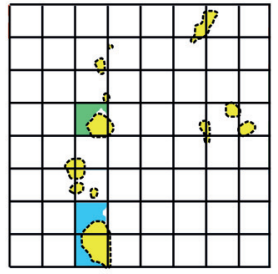

LWM frontal /semioval / parietal

FIG 1. A, The illustration shows axial T2-weighted and sagittal T7-weighted MR images. The pituitary-fastigium axis (dashed line) is used to position the grid (in red) with 64 voxels. B, An example of the WM, GM, and lesion PVE calculated on the MRSI grid and superimposed with the T2 image of the center of the slab. The yellow in the lesion PVE image represents the projection of the lesion content of the slab. C, The allocation of the different regions of GM, NAWM, and LWM based on the rows of the grid and the lesion content is seen.

estimate (PVE) for every tissue type in every voxel in the VOI (Fig $1 B)$.

\section{Metabolite Segmentation}

The absolute concentrations of the metabolites tNAA, tCr, Cho, mIns, and glutamate (Glu) were quantified for each voxel of the MRSI slab by using LCModel (http://www.lcmodel.com), ${ }^{20}$ as reported previously. ${ }^{17}$ Although the chemical shift artifacts are not very large at $1.5 \mathrm{~T}$, there are distinct differences between the first and last rows. They were therefore discarded in the analysis to avoid additional variation due to this effect (Fig $1 A$ ).

A segmentation based on existing methods ${ }^{21,22}$ was then applied to determine the specific metabolite concentrations for each tissue type and region. This approach accounts for the partial volume effects of GM, WM, and CSF by using the respective PVE values of the MRSI voxels and accounts for change in brain volume with time. Therefore, the variation of the concentrations is reduced by the distinction between WM and GM contributions. Moreover, inaccurate repositioning of the MRSI slab is partially compensated by the method, correcting for the change in the tissue content of every voxel. ${ }^{21-23}$ On the basis of the PVE, we determined 3 tissue types: GM, NAWM, and LWM. NAWM was defined as the WM content of voxels that contain $<1 \%$ lesion
PVE, whereas LWM was defined as the WM volume of voxels that contain $>5 \%$ lesion PVE. The WM of voxels containing a lesion PVE between $1 \%$ and $5 \%$ was discarded for the sake of specificity. Metabolite concentrations in the CSF were considered negligible. The MRSI slab was divided in 3 regions (frontal, centrum semiovale, and parietal) to take into account regional differences in concentrations (Fig 1C). ${ }^{21}$ Metabolite concentrations were then extrapolated for the 3 tissue types in each region (see the On-line Appendix for details).

The quality of the spectral acquisition was assessed with the average SNR and full width at half maximum over the 64 voxels for every subject and measurement, as estimated by LCModel. Spectra representing the quality of the acquisition have been published previously. ${ }^{17}$ We excluded the 2D-MRSI acquisition when the whole-grid average of the full width at half maximum exceeded 2 SDs above the group mean value.

The conservation of the metabolite quantities through the segmentation was verified with the concentration conservation factor (On-line Appendix).

\section{Statistical Analysis}

Multilevel modeling was performed with MLwiN, Version 2.18 (http://www. bristol.ac.uk/cmm/software/mlwin); the threshold for significance was $P=$ .05. To longitudinally assess any metabolite change with time, we applied a multilevel model with random intercepts and slopes to the data for each metabolite and tissue type (LWM, NAWM, GM). The results of the analysis were Bonferroni-corrected for multiple comparisons across tissue types; then, they were multiplied by 3 (or 2 for healthy controls without LWM). The model included 3 levels: 1) subject, 2) time point (baseline, month 6, month 12, month 18), and 3 ) region (frontal, centrum semiovale, parietal). The longitudinal rate of metabolite change was assessed by random slopes associated with the number of days elapsed since the baseline visit. Age, sex, group, and regions were included as covariates in this model. Additionally, mean lesional PVE was added as a covariate for the LWM model only.

For the baseline characterization, a multilevel analysis was performed for every metabolite including age and sex as covariates and including an interaction term between tissue region and group to assess the baseline difference between NAWM and LWM in both patient groups.

In the tissue types revealing no time evolution, we performed a cross-sectional analysis including all the time points as repeated measures, comparing patients receiving natalizumab with healthy controls. This was done with a multilevel analysis for every me- 
tabolite and tissue, with age and sex as covariates, with an interaction term between group and region that tests regional group differences for all time points. The significance of the effects was assessed with a Wald test and was Bonferroni-corrected for multiple-tissue-type comparisons.

\section{RESULTS}

\section{Subjects}

The groups did not differ in age and sex. Additionally, the patient groups did not differ in disease duration, disability (Expanded Disability Status Scale), brain volumes, and T2 lesion volume at baseline (Table 1). No serious or unanticipated adverse events attributed to MS medication developed in the patient groups.

Four MRSI acquisitions ( 1 of a patient receiving natalizumab, 2 of patients receiving IFNb/GA, and 1 of a healthy control) displayed insufficient spectral quality and were excluded from analysis.

\section{Lesion and Brain Volumes}

The patient groups did not differ on T2 lesion volumes at baseline $(P=.730$; Table 1$)$. No change in lesion volume in the VOI (the MRSI slab) was observed with time in the patients receiving natalizumab or in those receiving IFNb/GA (Fig 2, bottom row). Whole-brain normalized lesion volumes increased with time in the patients receiving IFNb/GA $(+0.47 \pm 0.15 \mathrm{~mL} /$ year, $P=$ $.002)$, whereas there was no significant change for the patients receiving natalizumab $(+0.32 \pm 0.33 \mathrm{~mL} /$ year, $P=.33)$.

The evolution of normalized total white matter and gray matter volumes and normalized whole-brain volumes of part of this cohort was presented in a separate study, showing reduced normalized whole-brain white matter during 12 months in both patient groups (O.T.W. et al, unpublished data, 2015).

\section{Baseline Metabolite Concentrations in LWM and Corresponding NAWM}

At baseline, patients receiving natalizumab had lower tNAA for all 3 LWM regions (between $10 \%$ and $16 \%, P<.008$ ), lower tCr for 2 LWM regions $(10 \%$ for frontal and $11 \%$ for semiovale, $P=$ .006), lower Glu for 3 LWM regions (between $12 \%$ and 15\%, $P<$ $.049)$, and higher mIns in the parietal LWM $(10 \%, P=.008)$ compared with the corresponding regional NAWM (all comparisons were corrected for age and sex in the model; for absolute concentrations, see Table 2). Reduced tNAA and tCr and increased mIns in LWM compared with corresponding NAWM were also seen in patients receiving IFNb/GA. This finding was significant for LWM semiovale tNAA $(14 \%, P<.001)$ and $\mathrm{tCr}$ $(9 \%, P=.02)$ and for mIns in frontal LWM $(18 \%, P<.001)$.

\section{Metabolite Evolution with Time}

Only patients receiving natalizumab showed metabolite changes with time and an increase in only LWM (frontal, centrum semiovale, and parietal LWM combined) for tNAA, tCr, and Glu. The estimated increase in LWM for tNAA was $0.51 \pm 0.13 \mathrm{mmol} / \mathrm{L} /$ year $(7 \%, P<.001)$; for $\mathrm{tCr}$, it was $0.26 \pm 0.11 \mathrm{mmol} / \mathrm{L} /$ year $(6 \%$, $P=.045)$; and for Glu, it was $0.49 \pm 0.19 \mathrm{mmol} / \mathrm{L} /$ year $(10 \%, P=$ .033) (Fig 2).

All metabolite concentrations remained statistically stable in the LWM of patients receiving IFNb/GA. Additionally, no concentration change was detected with time in NAWM or in GM in any group for any metabolite.

\section{NAWM and GM Metabolite Concentrations}

NAWM and GM were analyzed cross-sectionally in patients receiving natalizumab, because these regions did not evolve with time, including all the time points as repeated measures (Table 2).

We observed lower NAWM tNAA compared with healthy controls in parietal regions $(-7 \%, P=.043)$, with a trend in frontal regions $(-7 \%, P=.079)$. Higher concentrations of mIns were found in patients receiving natalizumab for all 3 NAWM regions compared with healthy controls (approximately 22\%, $P<$ $.001)$. No difference was found in GM for any metabolite between patients receiving natalizumab and healthy controls.

\section{Quality Parameters}

For the MRSI datasets, which were included in the analysis, the mean full width at half maximum and SNR were respectively $5.12 \pm 0.65 \mathrm{~Hz}$ and $10.9 \pm 1.7$ for the patients receiving natalizumab, $5.15 \pm 0.67 \mathrm{~Hz}$ and $10.7 \pm 1.2$ for those receiving IFNb/ $\mathrm{GA}$, and $5.10 \pm 0.68 \mathrm{~Hz}$ and $12.0 \pm 1.7$ for the healthy controls.

\section{DISCUSSION}

In patients with RRMS treated with natalizumab, we observed an increase of the tNAA concentration in LWM (7\%/year) combined with an increase of $\mathrm{tCr}$ (6\%/year) and Glu (10\%/year) during 18 months of treatment, while lesion volumes remained stable. In contrast, there were no metabolite changes with time in the LWM of patients continuing IFNb/GA, while whole-brain normalized lesion volumes increased in this group (however, there was no change with time in lesion volume in the VOI [the MRSI slab] in the patients receiving IFNb/GA).

The longitudinal increase of tNAA, tCr, and Glu in the LWM of patients treated with natalizumab, independent of change in lesion volume, might be a sign of enhanced mitochondrial axonal metabolism ${ }^{24}$ elicited by the anti-inflammatory effect of natalizumab. This effect is especially relevant for tNAA, which is synthesized in neuronal mitochondria. Higher levels of tNAA might, therefore, reflect increased mitochondrial activity. Recent studies have found that reactive oxygen species and reactive nitrogen species cause mitochondrial dysfunction and energy failure, which might be a mechanism for neuronal degeneration. ${ }^{25}$ By prevention of reactive oxygen species and reactive nitrogen species producing macrophages in the CNS, natalizumab might indirectly enhance mitochondrial axonal metabolism. Additionally, in animals models, axon damage has already been shown to be reversible by neutralization of reactive oxygen species and reactive nitrogen species. ${ }^{26}$ Our results corroborate a previous study that found that natalizumab suppressed the evolution of enhancing (active) lesions into T1-hypointense lesions, thereby preventing more severe axonal loss. ${ }^{27}$ This interpretation is in accordance with a study using diffusion tensor imaging that found an increase of fractional anisotropy in initially enhancing lesions after 12 months of natalizumab treatment. ${ }^{28}$

There are only a few pharmacologic MR spectroscopy studies in patients with RRMS, and 2 of these followed metabolite inten- 


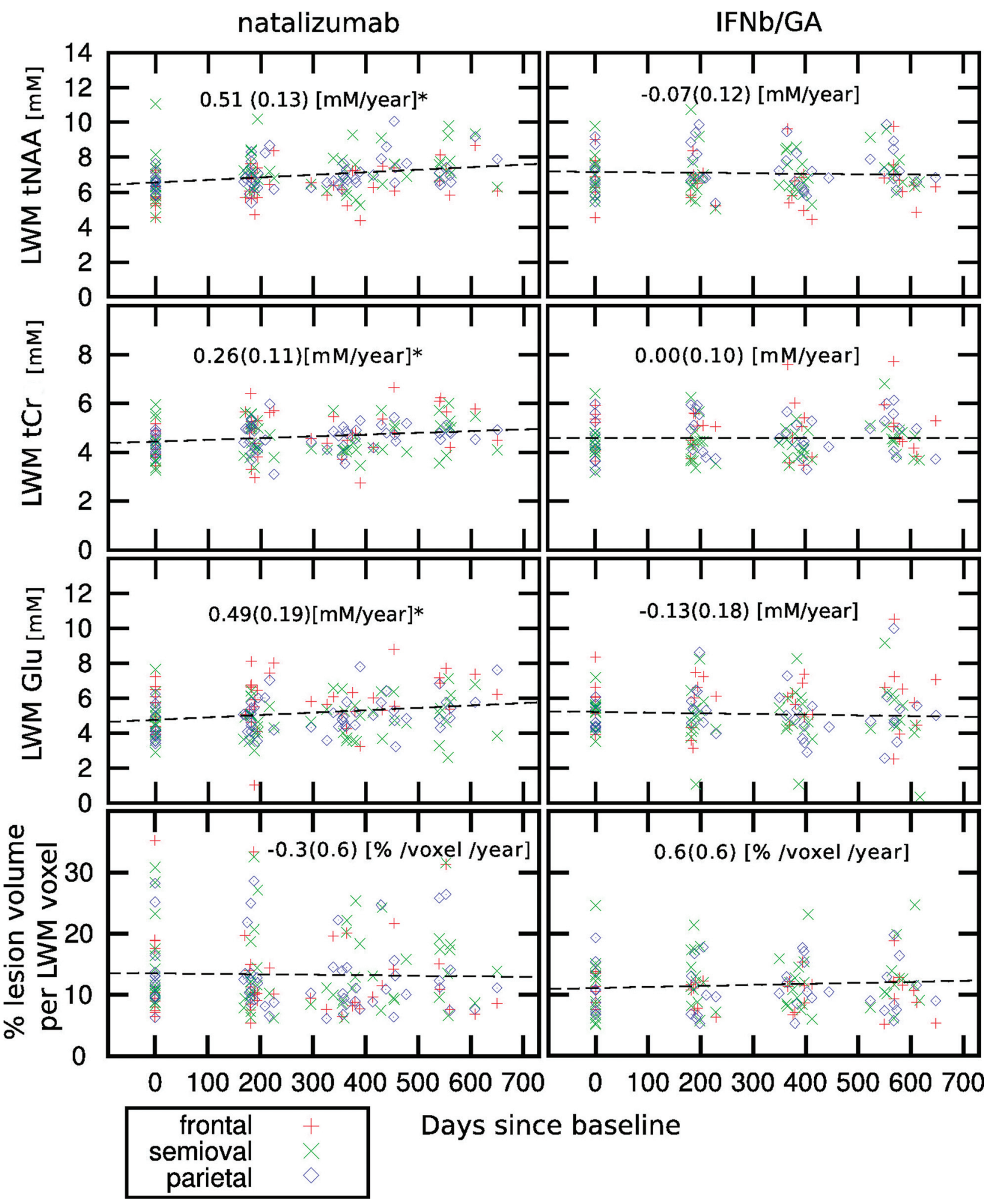

FIG 2. Scatterplot of individual measurements of $t N A A, t C r$, and Glu in LWM (mmol/L) (upper 3 rows) and percentage of lesion volume per LWM voxel (lower row) as a function of the days elapsed since the baseline for the patients treated with natalizumab and IFNb/GA. The asterisk indicates that patients treated with natalizumab show a significant increase of tNAA, tCr, and Glu in the LWM (mmol/L/year \pm SD). At each time point (baseline, month 6 , month 12, and month 18), the number of subjects in the natalizumab group was, respectively, 18, 25, 23, and 15; in the IFNb/GA group, it was 15, 17, 18, and 16; and in the healthy control group, it was 11, 12, 11, and 8.

sity ratios in a $2 \mathrm{D}$ section positioned in the center of the brain. ${ }^{14,15}$ One study investigating GA treatment in 18 patients with RRMS, compared with 4 untreated patients with RRMS, found a 7.1\% 1120 Wiebenga Jun 2015 www.ajnr.org increase in NAA/Cr in the GA-treated group during 24 months in NAWM. ${ }^{14}$ Another study in 10 patients with RRMS found a 5.5\% increase of NAA/Cr among patients treated with IFNb during a 
Table 2: Baseline absolute metabolite concentrations in NAWM, LWM, and GM ${ }^{\mathrm{a}}$

\begin{tabular}{|c|c|c|c|c|c|c|c|c|}
\hline & \multicolumn{3}{|c|}{ Natalizumab $(n=25)$} & \multicolumn{3}{|c|}{ IFNb/GA $(n=18)$} & \multicolumn{2}{|c|}{ Controls ( $n=12$ ) } \\
\hline & NAWM & LWM & GM & NAWM & LWM & GM & WM & GM \\
\hline \multicolumn{9}{|l|}{$\mathrm{tCr}$} \\
\hline Frontal & $4.86 \pm 0.71$ & $4.35^{c} \pm 0.58$ & $8.70 \pm 1.89$ & $4.93 \pm 0.92$ & $4.95 \pm 0.90$ & $8.70 \pm 1.41$ & $4.93 \pm 0.64$ & $8.48 \pm 1.31$ \\
\hline Semiovale & $4.67 \pm 0.63$ & $4.30^{c} \pm 0.81$ & & $4.83 \pm 0.75$ & $4.41^{b} \pm 0.77$ & & $4.72 \pm 0.60$ & \\
\hline Parietal & $4.52 \pm 0.62$ & $4.42 \pm 0.52$ & $7.78 \pm 0.88$ & $4.72 \pm 0.69$ & $4.57 \pm 0.93$ & $8.24 \pm 1.02$ & $4.58 \pm 0.45$ & $7.48 \pm 0.99$ \\
\hline \multicolumn{9}{|l|}{ tNAA } \\
\hline Frontal & $7.17 \pm 1.20$ & $5.89^{c} \pm 0.81$ & $11.1 \pm 1.74$ & $7.06 \pm 1.16$ & $6.75 \pm 1.54$ & $10.93 \pm 1.61$ & $7.62 \pm 0.96$ & $11.66 \pm 1.37$ \\
\hline Semiovale & $7.96 \pm 0.87$ & $6.70^{C} \pm 1.51$ & & $8.25 \pm 1.56$ & $7.05^{c} \pm 1.17$ & & $8.51 \pm 0.94$ & \\
\hline Parietal & $7.43^{d} \pm 1.05$ & $6.55^{c} \pm 0.74$ & $10.51 \pm 1.08$ & $7.59 \pm 0.98$ & $7.08 \pm 1.24$ & $11.52 \pm 1.46$ & $8.22 \pm 0.81$ & $10.89 \pm 1.30$ \\
\hline \multicolumn{9}{|l|}{ Cho } \\
\hline Frontal & $1.50 \pm 0.20$ & $1.51 \pm 0.25$ & $2.16 \pm 0.49$ & $1.59 \pm 0.28$ & $1.64 \pm 0.29$ & $2.11 \pm 0.45$ & $1.62 \pm 0.25$ & $2.08 \pm 0.39$ \\
\hline Semiovale & $1.44 \pm 0.19$ & $1.42 \pm 0.28$ & & $1.53 \pm 0.21$ & $1.49 \pm 0.28$ & & $1.49 \pm 0.16$ & \\
\hline Parietal & $1.13 \pm 0.24$ & $1.36 \pm 0.19$ & $1.31 \pm 0.22$ & $1.34 \pm 0.23$ & $1.39 \pm 0.27$ & $1.36 \pm 0.24$ & $1.37 \pm 0.17$ & $1.13 \pm 0.20$ \\
\hline mlns & & & & & & & & \\
\hline Frontal & $4.82^{\mathrm{e}} \pm 1.18$ & $5.29 \pm 1.01$ & $7.49 \pm 1.57$ & $4.44 \pm 0.92$ & $5.45^{c} \pm 1.51$ & $7.30 \pm 1.11$ & $3.90 \pm 0.65$ & $7.00 \pm 1.16$ \\
\hline Semiovale & $4.47^{e} \pm 1.18$ & $4.76 \pm 0.91$ & & $4.27 \pm 0.69$ & $4.71 \pm 1.01$ & & $3.56 \pm 0.44$ & \\
\hline Parietal & $4.79^{e} \pm 0.64$ & $5.59^{b} \pm 1.07$ & $6.47 \pm 0.91$ & $4.98 \pm 0.93$ & $5.32 \pm 1.42$ & $6.47 \pm 0.83$ & $4.01 \pm 0.62$ & $5.85 \pm 0.52$ \\
\hline \multicolumn{9}{|l|}{ Glu } \\
\hline Frontal & $6.34 \pm 1.46$ & $5.36^{\mathrm{b}} \pm 1.13$ & $12.96 \pm 2.98$ & $5.86 \pm 1.18$ & $6.03 \pm 1.48$ & $12.71 \pm 2.57$ & $6.25 \pm 0.96$ & $14.01 \pm 2.40$ \\
\hline Semiovale & $5.38 \pm 1.08$ & $4.73^{b} \pm 1.16$ & & $5.50 \pm 0.91$ & $5.11 \pm 0.96$ & & $5.49 \pm 0.72$ & \\
\hline Parietal & $5.17 \pm 1.34$ & $4.22^{b} \pm 0.94$ & $12.99 \pm 3.87$ & $5.37 \pm 1.57$ & $4.80 \pm 0.68$ & $12.68 \pm 1.44$ & $5.31 \pm 0.67$ & $11.81 \pm 1.85$ \\
\hline
\end{tabular}

${ }^{a}$ In mmol/L tissue, mean \pm SD. Absolute metabolite concentrations (mmol/L) of NAWM, GM, WM, and LWM in the frontal, centrum semiovale, and parietal regions averaged over the subjects at the baseline measurement.

b Significant difference within the group between NAWM and LWM in the same region of the baseline measurement $(P<.05)$.

' Significant difference within the group between NAWM and LWM in the same region of the baseline $(P<.01)$.

${ }^{\mathrm{d}}$ Significant differences between patients treated with natalizumab and healthy controls including all the time points $(P<.05)$.

e Significant differences between patients treated with natalizumab and healthy controls including all the time points $(P<.01)$.

treatment period of 12 months, compared with 6 untreated patients, in the entire $2 \mathrm{D}$ section. ${ }^{15}$ Whether the increases in NAA/Cr in the studies mentioned above are caused by increased NAA or reduced Cr remains unclear. The results of studies assessing metabolite ratios are difficult to compare with studies quantifying absolute concentrations. Because the presumed stability of tCr is questioned ${ }^{6}$ and has to be further investigated, the study of absolute concentrations, as in this study, is more enlightening. We did not observe any change in tNAA and tCr in NAWM in patients receiving IFNb/GA; this might be explained by differences in demographics and methodology.

No change with time was found in metabolite concentrations in NAWM and GM in both patient groups or in the healthy controls. In agreement with our results, a previous pharmacologic MR spectroscopy study that followed the effect of natalizumab on absolute metabolite concentrations in NAWM by using singlevoxel spectroscopy did not find evolution of any metabolite during 12 months in 27 patients with RRMS. ${ }^{29}$ During a longer follow-up time, we would speculate that further enhanced effects of natalizumab on metabolite evolution in NAWM might be seen, given the slow rate of tNAA change with time of NAWM in patients with RRMS.

At baseline, significantly lower tNAA, tCr, and Glu was found in the LWM of frontal, parietal, and centrum semiovale WM (except for parietal tCr) compared with corresponding NAWM in the patients receiving natalizumab. Lower tNAA, tCr, and Glu were also found in the LWM of patients receiving IFNb/GA, which was only significant for tNAA and $\mathrm{tCr}$ in the semiovale LWM. The reduced concentration of tNAA found in the current study in LWM compared with NAWM in patients at baseline is in line with that in previous studies. ${ }^{6}$ We found lower tCr in LWM, where conflicting results about tCr have been published. ${ }^{6}$ While in acute lesions, elevated Glu concentrations have been found, possibly due to inflammatory infiltrates of leukocytes, macrophages, and glial cells, ${ }^{30}$ lower Glu concentrations in LWM have, to our knowledge, never been described before. Several studies reported elevated concentrations of Cho in LWM, ${ }^{3,31}$ which were not observed in the current study. A possible explanation for these conflicting results could be the heterogeneity of the groups across studies. Some surveys specifically investigated active and/or chronic lesions. The lesion types were not differentiated in this study.

The metabolite segmentation method we used, in which we account for the tissue compartments of every voxel, is advantageous because most voxels contain different tissue types and because NAWM, GM, and LWM are known to differ in metabolite concentrations. ${ }^{6}$ Differences in metabolite concentrations also exist between frontal and parietal tissue, especially for GM. ${ }^{32}$ Higher concentrations are found in GM compared with WM, and mismatch in repositioning could have a great impact on the signal of voxels containing mixed tissues. ${ }^{33,34}$ With the current methodology, the extracted concentrations of GM, NAWM, or LWM are independent of the individual voxel composition. The results are, therefore, less affected by inaccuracies in section repositioning and intersubject anatomic differences in comparison with a voxel-by-voxel analysis method. The quality parameters full width at half maximum and SNR were in line with those in a previous reproducibility study in healthy controls, indicating reliable measurements in patients with MS. ${ }^{17}$

The multilevel mixed model used for the statistical analysis provides greater power than a 2-way ANOVA and can cope with nonorthogonal predictors and missing values. The approach used is particularly conservative by correcting the results of the regres- 
sion models by the number of tissues ( 3 for the patient groups and 2 for the healthy controls).

Apart from the relatively short follow-up time and the small sample size, the nonrandomized study design is a limitation of the study. Because natalizumab is second-line therapy at our center, only patients with active and breakthrough disease can receive treatment with natalizumab. As a result, these patients are more likely to have enhancing (active) lesions, compared with patients treated with IFNb/GA, who are relatively stable. Patients treated with natalizumab could possibly show higher normalization of LWM compared with LWM of stable MS; therefore, a direct comparison of the metabolite concentrations between the MS groups cannot be made. Unfortunately, we do not have gadolinium scans available for all patients; hence, the number of enhancing lesions between patient groups could not be compared. Nevertheless, there was no baseline difference in lesion volume, brain volume, and other disease characteristics such as disease duration and Expanded Disability Status Scale score, suggesting comparable disease severity of the patient groups.

The MRSI methodology used in this study might be especially informative in monitoring therapeutic effects with drugs specifically targeting neurodegeneration. This can mainly be expected at higher field strengths, which may allow a distinction between glutamate and glutamine, and which, due to a higher SNR, may allow a higher spatial resolution of the MRSI grid. It is recommended that additional quantitative MR imaging methods also be acquired, such as DTI or magnetic transfer ratio, to gain additional information about myelin integrity with time.

\section{CONCLUSIONS}

The possible beneficial effect of natalizumab on axonal functioning in patients with RRMS was assessed by using MRSI because natalizumab has a substantial clinical response. Patients treated with natalizumab showed an increase in tNAA, tCr, and Glu in lesional white matter. No change in NAWM and GM was found in any group for any metabolite. The increasing metabolite concentrations in LWM might be a sign of enhanced axonal metabolism. These findings may aid in explaining the large clinical effect of natalizumab. Larger studies with longer follow-up time are needed to assess the long-term effect of natalizumab on metabolite concentrations in LWM and NAWM and to monitor clinical evolution.

Disclosures: Oliver T. Wiebenga—RELATED: Grant: Dutch MS Research Foundation,* Biogen Idec.* Antoine M. Klauser-RELATED: Grant: Multiple Sclerosis Scientific Research Foundation.* Menno M. Schoonheim—UNRELATED: Consultancy: Genzyme*; Grants/Grants Pending: Dutch MS Research Foundation, ${ }^{*}$ Comments: Research supported by grant 08-650; Payment for Lectures (including service on Speakers Bureaus): Novartis, ${ }^{*}$ Serono, ${ }^{*}$ Comments: Postgraduate education on MS. Martin D. Steenwijk—RELATED: Grant: Dutch MS Research Foundation*; UNRELATED: Grants/Grants Pending: Dutch MS Research Foundation, Comments: Research sponsored through a program grant to the VU University Medical Center/MS Center Amsterdam, grant number 09-358d. Gijsbert J.A. Nagtegaal-RELATED: Grant: Dutch MS Research Foundation*, Biogen Idec*; UNRELATED: Grants/Grants Pending: Merck Serono*; Travel/Accommodations/Meeting Expenses Unrelated to Activities Listed: Bayer Healthcare Pharamceuticals. * *Money paid to the institution.

\section{REFERENCES}

1. Compston A, Coles A. Multiple sclerosis. Lancet 2008;372:1502-17

2. Geurts JJ, Calabrese M, Fisher E, et al. Measurement and clinical effect of grey matter pathology in multiple sclerosis. Lancet Neurol 2012;11:1082-92

3. He J, Inglese M, Li BS, et al. Relapsing-remitting multiple sclerosis: metabolic abnormality in nonenhancing lesions and normal-appearing white matter at MR imaging: initial experience. Radiology 2005;234:211-17

4. van Walderveen MA, Barkhof F, Pouwels PJ, et al. Neuronal damage in T1-hypointense multiple sclerosis lesions demonstrated in vivo using proton magnetic resonance spectroscopy. Ann Neurol 1999; 46:79-87

5. Tartaglia MC, Narayanan S, DeStefano N, et al. Choline is increased in pre-lesional normal appearing white matter in multiple sclerosis. J Neurol 2002;249:1382-90

6. Caramanos Z, Narayanan S, Arnold DL. 1H-MRS quantification of tNA and tCr in patients with multiple sclerosis: a meta-analytic review. Brain 2005;128:2483-506

7. Geurts JJ, Reuling IE, Vrenken H, et al. MR spectroscopic evidence for thalamic and hippocampal, but not cortical, damage in multiple sclerosis. Magn Reson Med 2006;55:478-83

8. Vrenken H, Barkhof F, Uitdehaag BM, et al. MR spectroscopic evidence for glial increase but not for neuro-axonal damage in MS normal-appearing white matter. Magn Reson Med 2005;53:256-66

9. Bellmann-Strobl J, Stiepani H, Wuerfel J, et al. MR spectroscopy (MRS) and magnetisation transfer imaging (MTI), lesion load and clinical scores in early relapsing remitting multiple sclerosis: a combined cross-sectional and longitudinal study. Eur Radiol 2009;19:2066-74

10. Chard DT, Griffin CM, McLean MA, et al. Brain metabolite changes in cortical grey and normal-appearing white matter in clinically early relapsing-remitting multiple sclerosis. Brain 2002;125:2342-52

11. Yednock TA, Cannon C, Fritz LC, et al. Prevention of experimental autoimmune encephalomyelitis by antibodies against alpha-4-beta-1 integrin. Nature 1992;356:63-66

12. Miller DH, Soon D, Fernando KT, et al. MRI outcomes in a placebocontrolled trial of natalizumab in relapsing MS. Neurology 2007;68:1390-401

13. Polman $\mathrm{CH}$, O'Connor $\mathrm{PW}$, Havrdova E, et al. A randomized, placebo-controlled trial of natalizumab for relapsing multiple sclerosis. N Engl J Med 2006;354:899-910

14. Khan O, Shen $\mathrm{Y}$, Caon $\mathrm{C}$, et al. Axonal metabolic recovery and potential neuroprotective effect of glatiramer acetate in relapsing-remitting multiple sclerosis. Mult Scler 2005;11:646-51

15. Narayanan S, DeStefano N, Francis GS, et al. Axonal metabolic recovery in multiple sclerosis patients treated with interferon beta1b. J Neurol 2001;248:979-86

16. Polman CH, Reingold SC, Edan G, et al. Diagnostic criteria for multiple sclerosis: 2005 revisions to the "McDonald Criteria." Ann Neurol 2005;58:840-46

17. Wiebenga, OT, Klauser, AM, Nagtegaal, GJ, et al. Longitudinal absolute metabolite quantification of white and gray matter regions in healthy controls using proton MR spectroscopic imaging. $N M R$ Biomed 2014;27:304-11

18. Steenwijk MD, Pouwels PJ, Daams M, et al. Accurate white matter lesion segmentation by $\mathrm{k}$ nearest neighbor classification with tissue type priors (kNN-TTPs). Neuroimage Clin 2013;3:462-69

19. Chard DT, Jackson JS, Miller DH, et al. Reducing the impact of white matter lesions on automated measures of brain gray and white matter volumes. J Magn Reson Imaging 2010;32:223-28

20. Provencher SW. Estimation of metabolite concentrations from localized in vivo proton NMR spectra. Magn Reson Med 1993;30:672-79

21. Maudsley AA, Domenig C, Govind V, et al. Mapping of brain metabolite distributions by volumetric proton $\mathrm{MR}$ spectroscopic imaging (MRSI). Magn Reson Med 2009;61:548-59

22. Tal A, Kirov II, Grossman RI, et al. The role of gray and white matter segmentation in quantitative proton MR spectroscopic imaging. NMR Biomed 2012;25:1392-400 
23. Gasparovic C, Yeo R, Mannell M, et al. Neurometabolite concentrations in gray and white matter in mild traumatic brain injury: an 1H-magnetic resonance spectroscopy study. J Neurotrauma 2009; 26:1635-43

24. Ciccarelli O, Altmann DR, McLean MA, et al. Spinal cord repair in MS: does mitochondrial metabolism play a role? Neurology 2010; 74:721-27

25. Witte ME, Geurts JJ, de Vries HE, et al. Mitochondrial dysfunction: a potential link between neuroinflammation and neurodegeneration? Mitochondrion 2010;10:411-18

26. Nikić I, Merkler D, Sorbara C, et al. A reversible form of axon damage in experimental autoimmune encephalomyelitis and multiple sclerosis. Nat Med 2011;17:495-99

27. Dalton CM, Miszkiel KA, Barker GJ, et al. Effect of natalizumab on conversion of gadolinium enhancing lesions to T1 hypointense lesions in relapsing multiple sclerosis. J Neurol 2004;251: 407-13

28. Fox RJ, Cronin T, Lin J, et al. Measuring myelin repair and axonal loss with diffusion tensor imaging. AJNR Am J Neuroradiol 2011; 32:85-91
29. Mellergård J, Tisell A, Dahlqvist LO, et al. Association between change in normal appearing white matter metabolites and intrathecal inflammation in natalizumab-treated multiple sclerosis. PLoS One 2012;7:e44739

30. Srinivasan R, Sailasuta N, Hurd R, et al. Evidence of elevated glutamate in multiple sclerosis using magnetic resonance spectroscopy at 3 T. Brain 2005;128:1016-25

31. Mader I, Roser W, Kappos L, et al. Serial proton MR spectroscopy of contrast-enhancing multiple sclerosis plaques: absolute metabolic values over 2 years during a clinical pharmacological study. AJNR Am J Neuroradiol 2000;21:1220-27

32. Pouwels PJ, Frahm J. Regional metabolite concentrations in human brain as determined by quantitative localized proton MRS. Magn Reson Med 1998;39:53-60

33. Chard DT, McLean MA, Parker GJ, et al. Reproducibility of in vivo metabolite quantification with proton magnetic resonance spectroscopic imaging. J Magn Reson Imaging 2002;15:219-25

34. Kreis R. Issues of spectral quality in clinical $1 \mathrm{H}$-magnetic resonance spectroscopy and a gallery of artifacts. NMR Biomed 2004;17: $361-81$ 